SATURDAY, 06 JUNE 2020

Scleroderma, myositis and related syndromes

\section{SAT0305 \\ PERFORMANCE OF HIGH FREQUENCY ULTRASOUND IN THE ASSESSMENT OF SKIN INVOLVEMENT IN SYSTEMIC SCLEROSIS}

G. Lettieri ${ }^{1}$, V. Picerno ${ }^{2}$, D. Temiz Karadağ ${ }^{2,3}$, M. C. Padula², G. A. Mennillo ${ }^{2}$, A. Padula ${ }^{2}$, S. D'angelo ${ }^{2}$, G. Abignano ${ }^{2} .{ }^{1}$ Radiology Department, San Carlo Hospital, Potenza, Italy; ${ }^{2}$ Rheumatology Institute of Lucania (IReL), Rheumatology Department of Lucania, San Carlo Hospital, Potenza, Italy; ${ }^{3}$ Canakkale State Hospital, Rheumatology Clinic, Canakkale, Turkey

Background: The modified Rodnan skin score (mRSS) is the current gold standard for skin assessment in systemic sclerosis (SSc) both in clinical trials and practice. High frequency ultrasound (HFUS) has been suggested to offer a quantitative assessment of skin thickness in SSc by several studies, however results are inhomogeneous with regards to the machine used, number of imaged sites, as well as the various stages of skin involvement.

Objectives: Aim of this cross-sectional study was to compare performance of HFUS in the assessment of skin involvement in diffuse cutaneous SSc (dcSSc) patients, at different disease stages, as compared with healthy controls (HC).

Methods: Dorsal finger, hand, forearm and upper arm skin of consecutive dcSSc patients, at different disease stages, and of matched-HC were scanned bilaterally using HFUS. Two investigators, expert in MSK ultrasound, blinded to the clinical details, measured skin thickness using Esaote MyLab70 equipped with a $22 \mathrm{MHZ}$ probe. Clinical involvement was assessed by a blinded operator using the mRSS and results were compared with imaging data. Statistical analysis was performed using GraphPad Prism software V.7.0.

Results: A total of 704 HFUS images were obtained from 22 dcSSc patients [20 Female, mean age $49( \pm 11)$ years, 12 with $\leq 5$ years disease duration] and $22 \mathrm{HC}$ [20 Female, mean age $50.7( \pm 6.7)$ years]. Skin thickness was significantly higher in SSc patients than in $\mathrm{HC}$ at fingers $(p<0.0001)$ and hands $(\mathrm{p}<0.0001)$, while no significant difference was found at the forearms and upper arms $(p>0.05)$ HFUS showed a good discriminative ability between SSc and HC skin at fingers and hands (AUC 0.91, 0.81, 0.6 and 0.65 for fingers, hands, forearms and upper arms respectively). When analysing the subgroup of SSc patients with $\leq 5$ years disease duration, HFUS showed a slightly lower performance in discriminating between $\mathrm{SSc}$ without clinical skin involvement (site $\mathrm{mRSS}=0$ ) and $\mathrm{HC}$ (AUC 0.68, 0.57, 0.68 for hands, forearms and upper arms respectively). Mean HFUS skin thickness significantly correlated with $\mathrm{mRSS}$ at site of analysis (hand: $r=0.78, p=<0.0001$; forearm: $r=0.47, p=0.0013$; upper arm: $r=0.52, p=0.0003$ ) and total mRSS (hand: $r=0.53, p=0.0002$; forearm: $r=0.63, p<0.0001$; upper arm: $r=0.63, p<0.0001)$. No significant correlation was found between finger skin thickness and mRSS (both local and total, $p>0.05$ ). Interobserver reliability for skin thickness was good to excellent at all sites with intraclass correlation coefficient ranging between 0.79 and 0.94 .

Conclusion: HFUS of the skin is a reliable measure of skin involvement in SSc Studies with higher number of patients with different clinical features are needed to explore the potential of HFUS to discriminate between healthy and SSc skin, including sites at a preclinical stage of involvement.

Disclosure of Interests: None declared

DOI: 10.1136/annrheumdis-2020-eular.6373

\section{SAT0306 \\ SEMIQUANTITATIVE AND QUANTITATIVE ANALYSIS OF LUNG CT IN THE ASSESSMENT OF INTERSTITIAL LUNG DISEASE IN IDIOPATHIC INFLAMMATORY MYOPATHIES WITH A FOCUS ON ANTISYNTHETASE.}

S. Barsotti ${ }^{1}$, C. Roncella ${ }^{2}$, A. Valentini ${ }^{3}$, L. Cavagna ${ }^{4}$, R. Castellana ${ }^{2}$, E. Cioffi ${ }^{1}$, A. Tripoli ${ }^{1}$, D. Caramella ${ }^{5}$, B. Bartholmai ${ }^{6}$, R. Neri ${ }^{1}$, F. Falaschi ${ }^{2}$, C. Romei ${ }^{2}$, M. Mosca ${ }^{1} .{ }^{1}$ Rheumatology Unit - Pisa University Hospital, Pisa, Italy; ${ }^{2}$ II Radiology Unit - Pisa University Hospital, Pisa, Italy; ${ }^{3}$ Department of Radiology, Fondazione IRCCS Policlinico San Matteo, Pavia, Italy; ${ }^{4}$ Rheumatology Division, University and IRCCS Policlinico S. Matteo Foundation, Pavia, Italy: ${ }^{5}$ Department of Diagnostic and Interventional Radiology, University of Pisa, Pisa, Italy; ${ }^{6}$ Department of Radiology, Mayo Clinic, Rochester, United States of America

Background: Interstitial lung disease (ILD), is common in patients with idiopathic inflammatory myopathies (IIM) and strongly impact on patients' morbidity and mortality. Patients with anti-aminoacyl-transfer RNA-synthetases (anti-ARS) antibodies are associated with an increased risk of ILD.

Objectives: Defining the radiological characteristics of IIM patients, with special focus on serological groups, through qualitative, semiquantitative and quantitative analysis of lung $\mathrm{CT}$.
Methods: This was a prospective study conducted from 2016 to 2019. Ninety-eight IIM patients (35 men, 63 women) were included. Myositis specific autoantibodies (MSA) were assessed with Myositis Prophyle III (Euroimmune, Lubeck).

Each patient had a baseline CT; the total score of Warrick (WS) was obtained at semiquantitative analysis. The radiological scores ILD\% (interstitial lung disease \%) and PVRS\% (pulmonary vascular related structure) were the result of quantitative analysis in 61 patients (CALIPER). Pulmonary function tests (PFTs) included TLC\%, FVC\% and DLCO $\%$ (65 patients). The analysis was conducted in the whole group and divided in subgroups based on their MSA pattern: in particular anti-ARS (Group 1) and patients negative to MSA (Group 2) were analysed.

Results: Positive correlations between ILD \% and PVRS\% (Rho=0.916; $\rho=0.000$ ), WS and ILD\% (Rho=0.663; $\rho=0.000)$ and WS and PVRS\% (Rho=0.637; $\rho<0.001)$ were found.

The most relevant inverse correlations were found between ILD\% and DLCO\% (Rho=-0.590; $\rho=0.001$ ), PVRS\% and DLCO\% (Rho=-0.549; $\rho<0.001)$ and WS and DLCO\% (Rho=-0.471; $\rho<0.001)$.

Statistically significant higher values of WS, ILD\% and PVRS\% were found in Group 1 (WS=15, ILD\%=11 and PVRS\%=3.5), compared to Group 2 (WS=2.5 ILD\%=0.84 and PVRS\%=2.2). NSIP pattern resulted dominant represented in the two groups ( $80 \%$ Group 1, 75\% Group 2). No statistically significant differences of DLCO $\%, F V C \%$ and $\mathrm{TLCO} \%$ were found.

Conclusion: The inverse correlations between the radiological scores and the functional data TLC\% and DLCO\% $(\rho<0.001)$ confirm the role of lung CT in the clinical management of ILD in IIM patients, and may represent a promising tool for clinical trials. For the first time anti-ARS and serological negative patients were defined through qualitative, semiquantitative and quantitative analysis of lung CT. Further study should be conducted in order to define the prognostic value of the quantitative analysis of lung CT in the follow up of IIM patients.

Disclosure of Interests: None declared

DOI: 10.1136/annrheumdis-2020-eular.4189

\section{SAT0307 PROGRESSION OF SUBCLINICAL MYOCARDIAL INVOLVEMENT IN PATIENTS WITH SYSTEMIC SCLEROSIS}

D. Benfaremo ${ }^{1}$, G. Stronati ${ }^{2}$, L. Manfredi ${ }^{1}$, L. Zuliani ${ }^{1}$, A. Ferrarini ${ }^{1}$, C. Fischetti ${ }^{1}$, C. Dichiara ${ }^{1}$, F. Guerra ${ }^{2}$, A. Dello Russo ${ }^{2}$, A. Gabriellii . ${ }^{1}$ Università Politecnica delle Marche, Dipartimento di Scienze Cliniche e Molecolari, Ancona, Italy; ${ }^{2}$ Università Politecnica delle Marche, Dipartimento di Scienze Biomediche e Sanità Pubblica, Ancona, Italy

Background: Systemic sclerosis (SSc) is a progressive autoimmune disease affecting the skin as well as internal organs, including the heart. A few studies have identified a subclinical heart involvement in patients with no pulmonary hypertension. Changes in myocardial deformation are consistent with the idea of SSc-related cardiomyopathy as a primary condition affecting the heart globally through microvascular dysfunction and subsequent myocardial fibrosis.

Objectives: The aim of the present study is to describe the progression of myocardial deformation in patients with SSc and no overt cardiac disease.

Methods: Prospective longitudinal study enrolling consecutive SSc patients referred to the Clinica Medica, University Hospital 'Ospedali Riuniti', Ancona, Italy, from February 2016 to December 2018. All patients fulfilled the 2013 ACR/EULAR classification criteria for SSc. Patients with structural heart disease, heart failure, atrial fibrillation or pulmonary hypertension were excluded. Disease subset, antibodies pattern, cardiovascular risk factors and involvement of other organ systems were recorded for each patient. An echocardiographic exam was performed for all patients at baseline and during their follow-up evaluation. Standard and speckle-tracking derived variables for the systolic and diastolic function of the left ventricle (LV) and right ventricle (RV) were acquired. Speckle tracking analysis software (EchoPAC 13.0; GE Medical Systems, Milwaukee, USA) was used to assess the GLS of the left and right ventricle, excluding the ventricular septum from right ventricular GLS calculations.

Results: Seventy-two patients (68 females, age $56.6 \pm 15.4$ years) were enrolled. Common echocardiographic parameters of left and right systolic function were within normal range at baseline and did not change during follow-up. Mean GLS, however, worsened for both left (from $-19.8 \pm 3.5 \%$ to $-18.7 \pm 3.5 \%, p=.034$ ) and right ventricle (from $-20.9 \pm 6.1 \%$ to $-18.7 \pm 5.4 \%, \mathrm{p}=.013$ ) during a median follow-up of 20 months (1st-3rd quartile 12-24 months). The increased impairment registered in SSc patients was homogenous across endocardial layers (LV from $-22.5 \pm-3.9$ to $-21.4 \pm 3.9$, $\mathrm{p}=.041$; RV from $-24.2 \pm 6.2$ to $-20.6 \pm 5.9, \mathrm{p}=.001$ ), mesocardial layers (LV $-19.7 \pm 3.6$ to $-18.7 \pm 3.5, \mathrm{p}=.043$; RV from $-21.3 \pm 5.9$ to $-18.8 \pm 5.7, \mathrm{p}=.012$ ) and epicardial layers (LV from $-17.1 \pm 3.0$ to $-16.4 \pm 3.1, p=.112$, RV $-18.8 \pm 6.3$ to $-16.0 \pm 8.4, p=.035$ ), as well 